\title{
Photocurrent generation in a metallic transition-metal dichalcogenide
}

\author{
Naveed Mehmood, ${ }^{1}$ Hamid Reza Rasouli, ${ }^{1}$ Onur Çakıroğlu, ${ }^{2}$ and T. Serkan Kasırga ${ }^{1,2, *}$ \\ ${ }^{1}$ UNAM- National Nanotechnology Research Center and Institute of Materials Science and Nanotechnology, Bilkent University, \\ Ankara, Turkey 06800 \\ ${ }^{2}$ Department of Physics, Bilkent University, Ankara, Turkey 06800
}

(Received 9 January 2018; revised manuscript received 25 April 2018; published 9 May 2018)

\begin{abstract}
Photocurrent generation is unexpected in metallic 2D layered materials unless a photothermal mechanism is prevalent. Yet, typical high thermal conductivity and low absorption of the visible spectrum prevent photothermal current generation in metals. Here, we report photoresponse from two-terminal devices of mechanically exfoliated metallic $3 \mathrm{R}-\mathrm{NbS}_{2}$ thin crystals using scanning photocurrent microscopy (SPCM) both at zero and finite bias. SPCM measurements reveal that the photocurrent predominantly emerges from metal/ $\mathrm{NbS}_{2}$ junctions of the two-terminal device at zero bias. At finite biases, along with the photocurrent generated at metal/ $\mathrm{NbS}_{2}$ junctions, now a negative photoresponse from all over the $\mathrm{NbS}_{2}$ crystal is evident. Among our results, we realized that the observed photocurrent can be explained by the local heating caused by the laser excitation. These findings show that $\mathrm{NbS}_{2}$ is among a few metallic materials in which photocurrent generation is possible.
\end{abstract}

DOI: 10.1103/PhysRevB.97.195412

Photocurrent generation in semiconducting 2D layered materials is dominantly due to photothermal [1], photovoltaic effects [2,3] as well as excitation of nonlocal hot carriers [4-6]. In metals, these mechanisms typically do not result in photocurrent generation except in a few cases. Photothermal effects are generally not significant in metals because of typical high thermal conductivity and low absorption of the optical excitation. Moreover, optically excited electrons in metals would not have a measurable contribution to the large number of intrinsic electrons near the Fermi level when a bias is applied. In a few cases such as metallic carbon nanotubes [7], graphene $[4,8,9]$, and gold nanoparticle networks [10], photocurrent generation has been reported. Light-induced current generation in metallic 2D layered transition-metal dichalcogenide (TMDC) is unprecedented; thus, it is not clear what mechanism will be prevalent. Here, we investigate photoresponse of mechanically exfoliated thin niobium disulfide $\left(\mathrm{NbS}_{2}\right)$ crystals using scanning photocurrent microscopy (SPCM) as an exemplary metallic TMDC. $\mathrm{NbS}_{2}$ can be found in layered form both in hexagonal $(2 H)$ and rhombohedral $(3 R)$ polytypes. While both polytypes are metallic [11], only $2 \mathrm{H}-\mathrm{NbS}_{2}$ is superconducting [12-15]. Sketches of the $3 R$ structure are depicted in Figs. 1(a) and $1(\mathrm{~b})$.

We report photocurrent measurements on two-terminal $3 R$ $\mathrm{NbS}_{2}$ devices near or at room temperature. $\mathrm{NbS}_{2}$ flakes of various thicknesses are patterned using optical lithography followed by gold/chromium electrode deposition. These devices will be referred to as top contact (TC) devices. Figure 1(c) depicts the scanning laser beam as well as the electrical connection on a scanning electron microscope micrograph of a TC device. The same electrical connection configuration is used throughout the paper; the ground terminal is connected to the

*Corresponding author: kasirga@unam.bilkent.edu.tr left; the current preamplifier terminal is connected to the right contact (see Supplemental Material for further details [16]).

Optical image of a typical TC device (named TC-1) is shown in Fig. 2(a). Reflection and photocurrent maps obtained with a 532 -nm laser of power $P=85 \mu \mathrm{W}\left(\sim 11 \mathrm{~kW} \mathrm{~cm}^{-2}\right)$ focused to a diffraction-limited spot are given in Figs. 2(b) and 2(c), respectively. Resistance vs temperature $(R-T)$ measurement given in Fig. 2(d) from TC-1 shows an $\sim 21.5-\Omega$ resistance $(R)$ at room temperature. $R-T$ measurement clearly shows the metallic nature of the $\mathrm{NbS}_{2}$ flake. An atomic force microscopy (AFM) height trace gives the flake thickness as $\sim 180 \mathrm{~nm}$ (AFM measurement is provided in Supplemental Material [16]). The photocurrent map for TC-1 under zero bias reveals that the extremum current is generated at the junctions where $\mathrm{NbS}_{2}$ flake meets with the metallic contacts. We consider that the photocurrent in this device has a photothermal origin as the other mechanisms are less likely in metal/metal junctions.

Firstly, we study the SPCM results on the TC-1 device in more detail [Figs. 2(a)-2(e)]. Due to the Seebeck effect, the lattice temperature difference $\Delta T_{C}$ between the two terminals of the contacts will generate a thermoelectric electromotive force (emf), $V_{T}=-\Delta S_{\mathrm{Au} / \mathrm{NbS}_{2}} \Delta T_{C}$. Using the absolute Seebeck coefficients reported in the literature for $\mathrm{NbS}_{2}$ and $\mathrm{Au},-4$ and $\sim 2 \mu \mathrm{V} / \mathrm{K}$, respectively [17,18], the difference in the Seebeck coefficients of $\mathrm{NbS}_{2}$ and $\mathrm{Au}$ is $\Delta S_{\mathrm{Au} / \mathrm{NbS}_{2}}=S_{\mathrm{Au}}-S_{\mathrm{NbS}_{2}} \approx$ $-6 \mu \mathrm{V} / \mathrm{K}$. When the laser is focused at the junction, the maximum emf generated is $V_{\mathrm{PC}}=R I_{\mathrm{PC}} \approx 4.5 \mu \mathrm{V}$, where $I_{\mathrm{PC}}$ is the measured photocurrent. $I_{\mathrm{PC}}$ measured on the right and the left junctions are -130 and $150 \mathrm{nA}$, respectively. Comparing these $V_{\mathrm{PC}}$ values to $V_{T}$ shows that $\Delta T_{C} \approx 0.54 \mathrm{~K}(\approx 0.46 \mathrm{~K}$ for the left junction) for $P=85 \mu \mathrm{W}$, which corresponds to $6.3 \mathrm{mK} / \mu \mathrm{W}(5.5 \mathrm{mK} / \mu \mathrm{W}$ for the left junction). These values of $\Delta T_{C} / P$ are consistent with the values reported in similar studies in the literature $[1,19]$.

To test if we get a consistent behavior for different $P$ values, we parked the laser spot on the metal/ $\mathrm{NbS}_{2}$ junctions where 

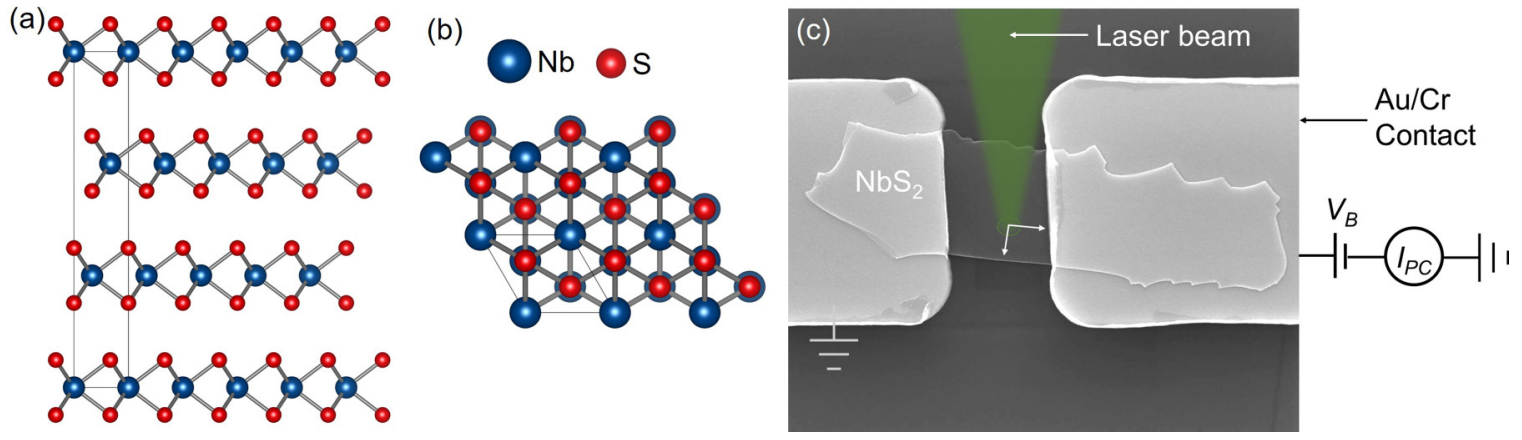

FIG. 1. (a) Crystal structure of $3 R-\mathrm{NbS}_{2}$ is depicted from the side and (b) from the top. (c) Measurement configuration is depicted on a scanning electron microscope micrograph of a two-terminal device. Laser beam raster scans over the sample with a diffraction-limited spot. Throughout the paper, left contact is grounded, and the bias is applied through the right contact using a current preamplifier.

the photocurrent is at its maximum and minimum, marked by yellow dashed circles in Fig. 2(c). Then, while simultaneously reading the laser power through a 50:50 beam splitter, we tuned the laser power with a variable neutral density filter and recorded the photocurrent. Figure 2(e), upper panel shows $V_{\mathrm{PC}}$ vs $P$. For both junctions we observe a similar linear change in the photoresponse with increasing laser power. Moreover, when we plot the calculated temperature increase per unit laser power vs the laser power [Fig. 2(e), lower panel], we see that at both junctions, $\Delta T_{C} / P$ has a similar value with a small difference. This difference in $\Delta T_{C} / P$ at the opposing junctions might be due to slight differences in the gold contact edges [Fig. 1(c)] as well as positioning of the laser spot. $\mathrm{NbS}_{2}$ crystals transferred on top of prepatterned gold contacts also exhibit a similar photoresponse to the TC devices as discussed later in the text.
When we apply bias to the device, while the photoresponse at the metal/ $\mathrm{NbS}_{2}$ junction changes, we now observe a photoresponse all over the crystal. Figures 3(a) and 3(b) show photocurrent maps of TC-1 taken under 50- and $-50-\mathrm{mV}$ biases $\left(V_{B}\right)$, and Fig. 3(c), upper panel shows the photoresponse along the center of the device at several finite biases. We calculate the photoconductance $G_{\mathrm{PC}}=\left(I_{B}-I_{0}\right) / V_{B}$, where $I_{0}$ is the zero-bias photocurrent and $I_{B}$ is the photocurrent at any given $V_{B} . G_{\mathrm{PC}}$ values for various biases are the same throughout the crystal [Fig. 3(c), lower panel]. The change in photoresponse due to the applied bias indicates a bias-independent photoconductance mechanism that can be explained by the laser heating as detailed in the following paragraph.

The photoresponse observed from all over the crystal under bias can be explained by the local temperature increase, $\delta T_{L}$, caused by the laser. As the temperature increases locally, the
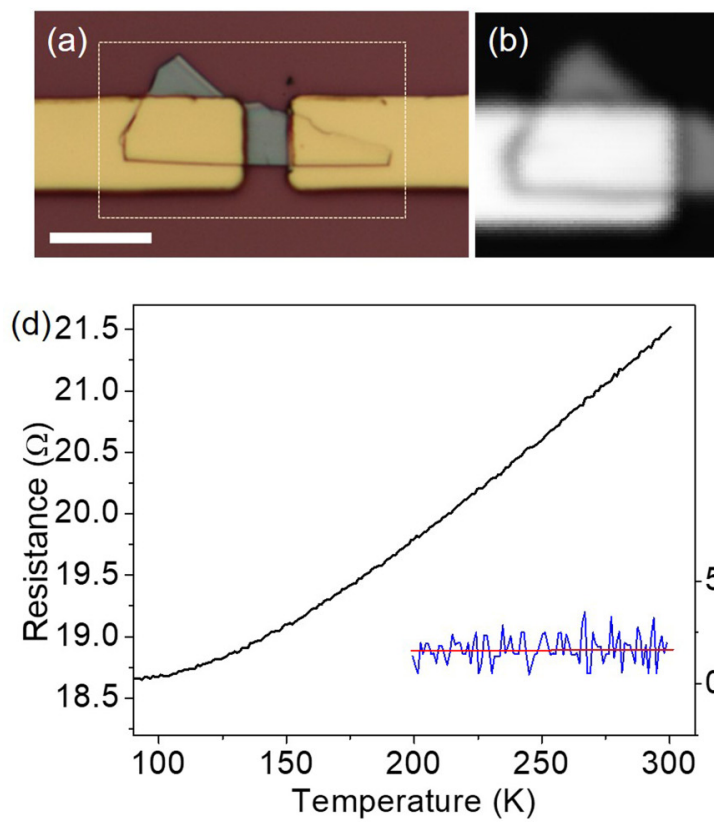
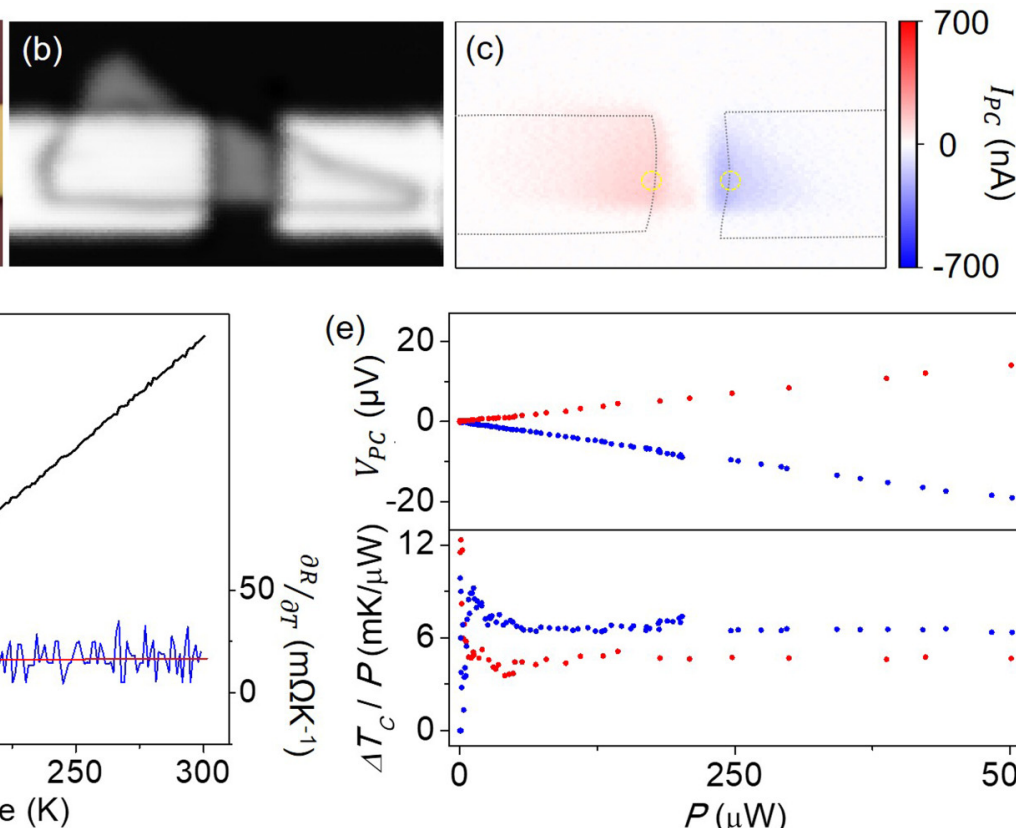

FIG. 2. (a) Optical microscope image of TC-1 is shown. SPCM measurement is taken from the region boxed with a dashed rectangle. Scale bar is $10 \mu \mathrm{m}$. (b) Reflection map and (c) the corresponding photocurrent map of TC-1 under zero bias shows photocurrent emerging around the contacts. Black dashed lines indicate the outlines of the contacts. (d) $R-T$ graph shows the metallic characteristic of the sample. $\partial R / \partial T$ is given in the inset from 200 to $295 \mathrm{~K}$ as a reference. (e) Upper panel shows $V_{\mathrm{PC}}$ vs $P$ measured from the metal/ $\mathrm{NbS}_{2}$ junctions [positions indicated by yellow dashed circles in (c)], red points from the left contact, and blue points from the right contact. Lower panel shows the calculated $\Delta T_{C} / P$ from the $V_{T}$ vs $P$ data. 


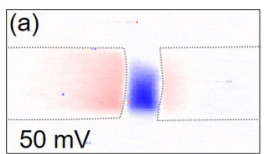

(b)
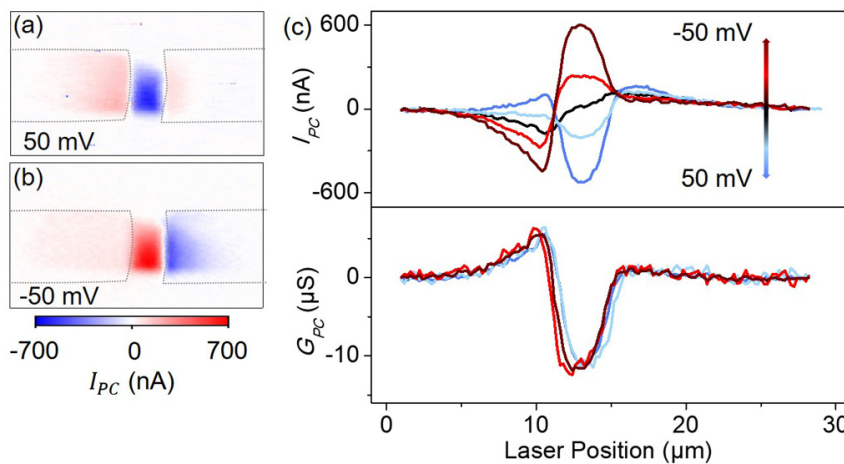

FIG. 3. (a) Photocurrent map of TC-1 under $50-\mathrm{mV}$ and (b) $-50-\mathrm{mV}$ bias is given. Photocurrent is generated all over the crystal between the contacts when the bias is applied. Dashed gray line represents the outline of the metal contacts. (c) Line trace along the center of the crystal shows the photocurrent (upper panel) under -50-, $-20-, 0-, 20-$, and 50-mV biases and respective photoconductances (lower panel). It is clear that the photoconductance $G_{\mathrm{PC}}$ is the same for all different biases.

electrical conductivity decreases on and around where the laser spot hits. This decrease leads to a negative photoconductance in such a way that the dc current due to the applied bias decreases. As the only determining parameter in the local resistivity change is the laser power, applied bias has no effect on the conductance. For this reason, the photoconductance is bias-independent. We calculated the change in the resistance of the device with the approximation that the temperature increase within a disk of diameter $D \approx 1 \mu \mathrm{m}$ is uniform, and outside the disk it is zero (see Supplemental Material for details [16]). For a device like TC-1, this calculation shows that for a laserinduced local temperature rise of $\delta T_{L} / P \approx 40 \mathrm{mK} / \mu \mathrm{W}$, the magnitude of the measured photocurrent is in excellent agreement with the calculated value. This local temperature change being slightly higher than the temperature increase extracted from the metal/ $\mathrm{NbS}_{2}$ junction, $\Delta T_{C} / P$, is consistent with the lower thermal conductivity of $\mathrm{SiO}_{2}$ substrate as compared to $\mathrm{Au} / \mathrm{Cr}$.

Negative photoconductance observed from the center of the crystal should diminish in a device with poor electrical contacts as the effective bias on the crystal ( $V_{\text {eff }}$ ) will be much lower due to the potential drop through the contact resistance, $R_{C}$. This is what we observe in crystals transferred on top of prepatterned thin metallic contacts. Figure 4(a) shows a bottom contact device (BC-1) with the $\mathrm{NbS}_{2}$ crystal ( 100-nm-thick) transferred using the polycarbonate transfer method [20] on top of 10/5-nm-thick Au/Cr contacts. The resistance of BC-1 is measured as $R=600 \Omega$ at room temperature. Firstly, the zerobias photocurrent map obtained with a 532-nm laser of power $P=32 \mu \mathrm{W}$ [Fig. 4(b)] shows a local photoresponse unlike TC devices. The magnitude of the zero-bias photoresponse is an order of magnitude smaller. This can be attributed to the higher resistance of the device. When we calculate the $\Delta T_{C} / P$ value for $\mathrm{BC}-1$ we get about $50 \mathrm{mK} / \mu \mathrm{W}$, which is higher as compared to TC devices. This is also expected as the thermal contact of $\mathrm{NbS}_{2}$ flake with the contact pads is poorer compared to TC devices.

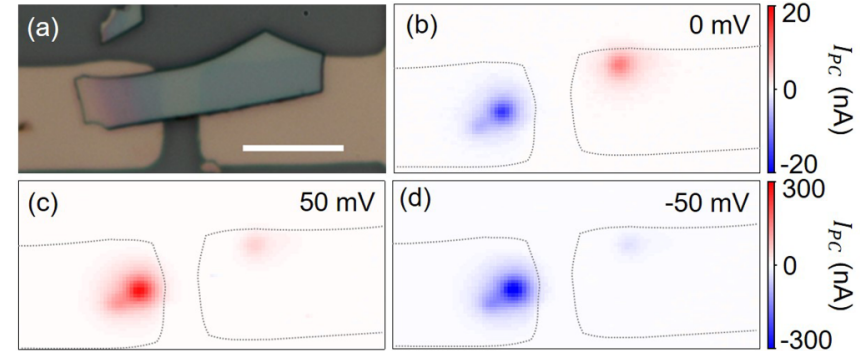

FIG. 4. (a) Optical microscope image of bottom contact device (BC-1) is shown. Scale bar is $10 \mu \mathrm{m}$. Photocurrent maps (b) under zero bias, (c) $50-\mathrm{mV}$, and (d) $-50-\mathrm{mV}$ bias show that there is no observable photoconductance change in the center of the crystal. This can be explained by the fact that the contact resistance of $\mathrm{BC}-1$ is so high that $V_{\text {eff }}$ on the crystal is small and hence local resistance change caused by photothermal heating becomes insignificant. Dashed gray line represents the outline of the metal contacts.

Based on our resistivity measurements on TC-1, a crystal like the one used in $\mathrm{BC}-1$ should have a resistance of $\sim 25$ $\Omega$ at room temperature. The difference between the expected resistance, $R_{E}$, and the measured $R$ can be attributed to the contact resistance. The photoresponse at zero bias is localized to small regions on the contacts and the strength of the photothermal current is different in amplitude for the ground and the preamp side. This is an indicator of varying contact quality across the contacts. When a finite bias is applied, only the signal coming from the $\mathrm{NbS}_{2} /$ metal junctions is altered and we do not observe a photoresponse throughout the crystal [Figs. 4(c) and 4(d)]. $V_{\text {eff }}$ on the crystal can be written as $V_{\text {eff }}=\left[V_{B} /\left(R_{E}+R_{C}\right)\right] R_{E}$. For BC-1, $V_{\text {eff }}$ is as low as $4 \%$ of $V_{B}$. Thus, photocurrent coming from the center of the crystal is less than a nanoampere in a device like BC-1.

The results we report in this work demonstrate that the light-induced current generation even in metallic TMDC is possible. SPCM measurements reveal the photothermal origin of the generated photocurrent and this result is consistent with the correlated nature of $3 R-\mathrm{NbS}_{2}$ crystals [21]. We would like to note that we performed SPCM experiments under vacuum to rule out any other possible photocurrent mechanisms due to oxygen in the ambient [22]. We observe no difference between the photoresponse of devices under vacuum or in ambient (Supplemental Material [16]). We also performed SPCM on indium contact devices from which photoresponse similar to TC devices has been observed (see Supplemental Material for details [16]). Finally, we would like to add that the polarization of the laser excitation has no effect on the photocurrent generation for both zero and finite-bias measurements. Experiments performed under linearly polarized and unpolarized light show no significant difference (see Supplemental Material [16]). This study shows that $3 R-\mathrm{NbS}_{2}$ sets an example to a few other metallic materials that photocurrent generation is possible. Our findings will be useful in engineering of all-TMDC optoelectronic components [23].

We would like to thank Engin Can Sürmeli, Koray Yavuz, Breera Maqbool, and Talha Masood Khan for their useful comments on the manuscript. This work is supported under TUBITAK 1001 program, Grant No. 214M109. 
[1] M. Buscema, M. Barkelid, V. Zwiller, H. S. van der Zant, G. A. Steele, and A. Castellanos Gomez, Nano Lett. 13, 358 (2013).

[2] L. Britnell, R. M. Ribeiro, A. Eckmann, R. Jalil, B. D. Belle, A. Mishchenko, Y. J. Kim, R. V. Gorbachev, T. Georgiou, S. V. Morozov et al., Science 340, 1311 (2013).

[3] M. M. Furchi, D. K. Polyushkin, A. Pospischil, and T. Mueller, Nano Lett. 14, 6165 (2014).

[4] N. M. Gabor, J. C. Song, Q. Ma, N. L. Nair, T. Taychatanapat, K. Watanabe, T. Taniguchi, L. S. Levitov, and P. Jarillo-Herrero, Science 334, 648 (2011).

[5] L. Wang, Z. Wang, H. Y. Wang, G. Grinblat, Y. L. Huang, D. Wang, X. H. Ye, X. B. Li, Q. Bao, A. S. Wee et al., Nat. Commun. 8, 13906 (2017).

[6] Q. H. Wang, K. Kalantar-Zadeh, A. Kis, J. N. Coleman, and M. S. Strano, Nat. Nanotechnol. 7, 699 (2012).

[7] M. Barkelid and V. Zwiller, Nat. Photonics 8, 47 (2013).

[8] J. C. Song, M. S. Rudner, C. M. Marcus, and L. S. Levitov, Nano Lett. 11, 4688 (2011).

[9] D. Sun, G. Aivazian, A. M. Jones, J. S. Ross, W. Yao, D. Cobden, and X. Xu, Nat. Nanotechnol. 7, 114 (2012).

[10] X. Ning Xie, Y. Xie, X. Gao, C. Haur Sow, and A. Wee, Adv. Mater. 21, 3016 (2009).

[11] M. Naito and S. Tanaka, J. Phys. Soc. Jpn. 51, 219 (1982).

[12] W. G. Fisher and M. J. Sienko, Inorg. Chem. 19, 39 (1980).

[13] J. Molenda, T. Bak, and J. Marzec, Phys. Status Solidi A 156, 159 (1996).
[14] D. R. Powell and R. A. Jacobson, J. Solid State Chem. 37, 140 (1981).

[15] Y. H. Huang, C. C. Peng, R. S. Chen, Y. S. Huang, and C. H. Ho, Appl. Phys. Lett. 105, 093106 (2014).

[16] See Supplemental Material at http://link.aps.org/supplemental/ 10.1103/PhysRevB.97.195412 for experimental methods for device fabrication, experimental setup, sample characterization results, photothermal photoconductance calculations, lowpressure SPCM measurements, laser power and polarizationdependent SPCM measurements, and measurements on various other devices.

[17] S. Zhao, T. Hotta, T. Koretsune, K. Watanabe, T. Taniguchi, K. Sugawara, T. Takahashi, H. Shinohara, and R. Kitaura, 2D Mater. 3, 025027 (2016).

[18] N. Cusack and P. Kendall, Proc. Phys. Soc. 72, 898 (1958).

[19] X. Xu, N. M. Gabor, J. S. Alden, A. M. van der Zande, and P. L. McEuen, Nano Lett. 10, 562 (2010).

[20] Y. C. Lin, C. Jin, J. C. Lee, S. F. Jen, K. Suenaga, and P. W. Chiu, ACS Nano 5, 2362 (2011).

[21] T. S. Kasirga, D. Sun, J. H. Park, J. M. Coy, Z. Fei, X. Xu, and D. H. Cobden, Nat. Nanotechnol. 7, 723 (2012).

[22] Y. H. Huang, R. S. Chen, J. R. Zhang, and Y. S. Huang, Nanoscale 7, 18964 (2015).

[23] H. Bark, Y. Choi, J. Jung, J. H. Kim, H. Kwon, J. Lee, Z. Lee, J. H. Cho, and C. Lee, Nanoscale 10, 1056 (2018). 